\title{
STRUCTURAL AND MODAL ANALYSIS IN SOLIDWORKS OF BASIC STRUCTURE OF EQUIPMENT TO PREPARE GERMINATIVE BED IN STRIPS
}

\author{
Gabriel Valentin Gheorghe, Catalin Persu, Iuliana Gageanu, Dan Cujbescu \\ National Institute of Research-Development for Machines and Installations Designed to Agriculture \\ and Food Industry, Romania \\ gabrielvalentinghe@yahoo.com, persucatalin@yahoo.com, \\ iulia.gageanu@gmail.com,dcujbescu@yahoo.com
}

\begin{abstract}
This article presents the way to obtain the structural model for elementary linear-elastic static analysis of equipment to prepare the germinative bed in strips main resistance structure. Also, to prove the functionality of the structural model obtained, the structural analysis results for the linear elastic static test are presented. These results are useful for estimating the safety factor and for assessing the behaviour in major overstress situations at the main part of the machine. Structure dynamics is a very broad discipline that uses a huge arsenal of theoretical and experimental methods to solve a fundamental problem of structures: the dynamic response to variable tasks over time. Vibrations, and especially vibration in resonance modes, are problems that occur frequently in large structures. As large structures with large numbers of components cannot be optimally engineered for resonant regimes, it is often done to resolve structures or improve them by using the modal analysis of the mathematical models of these structures. The usefulness of this analysis is particularly evident in the testing phase and even in the first stages of operation, when it is necessary to improve the working regime of a product of the type analyzed. The main results of the static linear-elastic structural analysis are: the values of the reactions in the holders, vector field distribution of the relative - resultant displacement in the structure, tensor fields' distribution of the specific deformation and the Cauchy stress tensor in the same structure. Also, an important result for the structure safety is the distribution of the safety factor. The analysis of the equipment's own spectrum allows proper identification of the main frequencies, at which a resonant working regime can occur.
\end{abstract}

Keywords: structural analysis, modal analysis, von Mises Stress, own frequencies.

\section{Introduction}

Cultivators, as well as harrows, are designed for surface soil works and have soil loosening and weed destruction as the main purpose. By purpose, the cultivators are divided into cultivators for total work, cultivators for soil work between rows and universal cultivators. Regardless of their intended purpose, mechanical traction cultivators consist of the following main assemblies and mechanisms: the frame, lifting and adjustment mechanism, driving mechanism, wheels, fastening devices for the active parts and the active parts themselves. Weeding cultivators (Fig. 1) are used to work between the rows of different industrial plants and oil seed crops, sown by sowing machines or planted by planters. Weeding cultivators are divided into trailed cultivators and carried cultivators [1].

Optimal design or improvement of a complex mechanical structure are activities that are currently carried out in the work of advanced companies working in the field of mechanical and other types of structures. Designing an optimal product (at least from some points of view) or optimizing the existing products requires complex working tools that are nowadays integrated into CAD-CAE complex programs. The workflow in the CAD-CAE complex is often fragmented, due to the great workload and complex knowledge it requires. For these reasons, in general, the CAD model may come from suppliers, who do not have the qualification to do the structural analysis and vice versa, CAE models are used by structuralists, who do not have all the engineering knowledge needed to create manufacturing drawings.

The usefulness of the own frequencies and own vibration modes calculation results from the fact that they can explain certain resonance working modes of external or internal origin. Working modes of external origin are less common. These may be caused by a possible periodic structure of the soil (highly improbable, but not impossible, if we refer, for example, to surfaces previously profiled for various cultures or for anti-erosion purposes), and/or by critical working speeds or even traction oscillations. Internal resonances may be caused by the oscillations of some components of the structure, on frequencies equal to or very close to one of the structure own frequencies. Internal resonances disturb the working regime, can affect the quality of the work or even lead to premature wear of the machine and the aggregate. The intense interaction with the tractor can induce the vibrations of the analyzed structure in the tractor resistance structure, eventually retransmitted by 
suspension to the tractor operator. Consequences can be estimated using the knowledge on vibration effects on the human body. The effects of vibrations on the work quality of the machines for soil processing are also a much discussed topic [2-6].

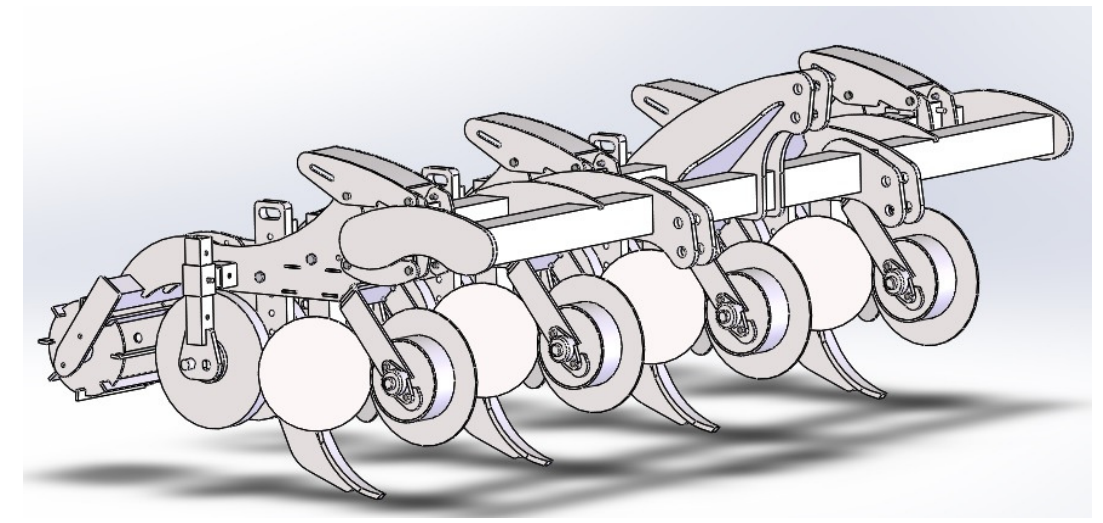

Fig. 1. Equipment to prepare germinative bed in strips

The traction resistance force of agricultural machines is given by the component parallel with the soil surface of the resultant of all the resistance forces that arise as a result of agricultural machines performing the working process.

In a general form, the sum of the traction resistance force of agricultural machines $R_{M}$ is expressed by the relation:

$$
R_{M}=R_{r}+R_{f}+R_{d}+R_{T} \pm R_{p}+R_{i}+R_{a}+R_{\varepsilon}
$$

where $R_{r}$ - rolling resistance force resulting from axles' friction in wheel bushings, friction of wheel rims with the soil surface and soil deformation by machine wheels;

$R_{f}$ - traction force generated by sliding friction of the working environment (soil, stems, seeds, fertilizers, etc.) on the surface of the machine active parts (plows, breasts, cutters, distribution devices etc.) and internal friction within the working environment, as a result of the active parts action on it;

$R_{d}$ - deformation resistance force of the processed material generated by the action of the active parts (deformation of the furrow during the plowing, soil loosening while harrowing and cultivator working, deformation of the soil caused by sowing machines, plows, cutting the weeds while hoeing and the stalks while harvesting);

$R_{\varepsilon}$ - resistance force caused by the transmission of kinetic energy to soil particles during plowing and field preparation for sowing, as well as other materials, during the working process of the machines;

$R_{T}$ - resistance force resulting from frictions in the transmission mechanisms;

$R_{p}$ - extra resistance force, when moving on slope lands;

$R_{i}$ - resistance force that occurs during uneven motion of the machines, because of inertial forces;

$R_{a}-$ air resistance force, when the machine is moving.

Relation (1) is used in the research activity in order to improve the machine construction and operation.

The resistance force to traction, when idling, is equal to the resistance force, when rolling $R_{r}$ and depends both on the construction parameters of the wheel and the nature and condition of the land, on which the vehicle is moving, being directly proportional to the rolling resistance coefficient of the agricultural machine wheels $\mathrm{f}_{\mathrm{m}}$ and the machine weight $G_{m}$ distributed to the wheels, namely:

$$
R_{r}=f_{m} \cdot G_{m}
$$

The traction resistance force of operating agricultural machines also includes the resistance force to traction when idling.

The traction resistance force of operating agricultural machines that are usually trailed is determined with the relation: 


$$
R_{m}=K \cdot B_{l},
$$

where $K$-specific resistance per meter of working width of the agricultural machine, in $\mathrm{N} \cdot \mathrm{m}^{-1}$;

$B_{l}-$ constructive working width of the machine, in $\mathrm{m}$.

The specific resistance $\mathrm{K}$ is determined experimentally. Its value is obtained by the ratio between the traction resistance force of the agricultural machines and the working width. For different agricultural machines, the indicative values of the specific resistance $\mathrm{K}$ are shown in Table 1 [7].

Indicative values of the specific resistance $K$ for different agricultural machines

\begin{tabular}{|c|c|}
\hline \multicolumn{1}{|c|}{ Machine type } & $\boldsymbol{K}, \mathbf{N} \cdot \mathbf{m}^{-\mathbf{1}}$ \\
\hline Rigid and articulated tooth harrows: & \\
$-\quad$ Light & $300-400$ \\
$-\quad$ Medium & $500-700$ \\
$-\quad$ Heavy & $800-1000$ \\
\hline Adjustable tooth harrows: & $700-1000$ \\
$-\quad$ On plowed soil & $550-850$ \\
\hline$\quad$ On sown soil & $900-1400$ \\
\hline Stellar harrows & \\
\hline Disc harrows: & $2000-3000$ \\
$-\quad$ Medium & $4000-8000$ \\
\hline Heavy & $1200-2800$ \\
\hline Rollers: & $600-1300$ \\
\hline - Flat & \\
\hline Cultivators equipped with: & $\mathbf{2 5 0 0 - 3 5 0 0}$ \\
$-\quad$ Chisels for loosening & $800-2000$ \\
\hline$\quad$ Arrow knives & \\
\hline
\end{tabular}

\section{Materials and methods}

In the first phase, using the formula (3), we calculate the resistance forces to be applied on the chisels in the SolidWorks program, Simulation, Static Simulation module.

$$
R_{m}=K \cdot B_{l},
$$

where $K=3500 \mathrm{~N} \cdot \mathrm{m}^{-1}$;

$$
B_{l}=l \cdot n,
$$

where 1 - working width of a chisel, m;

$\mathrm{n}$ - number of working parts;

$B_{l}=0.4 \cdot 4=1.6 \mathrm{~m}$;

$R_{m}=3500 \cdot 1.6=5600 \mathrm{~N}$.

The analyzed structure is a product in the design phase. After the described transformations, we obtained a common CAD model within SolidWorks. The sub-assembly in Figure 1, on which the structural analysis and modal analysis will be performed, is composed of 337 benchmarks.

After fixing the equipment in the three points (Figure 2) the calculated forces are applied and the meshing of the structure is made, resulting in a mesh consisting of 77980 knots and 251363 elements.

When transforming the CAD SolidWorks model into the CAE SolidWorks model, due to the initial model, an assembly resulted that could not be used to perform the structural analysis. To solve this impediment, we isolated each benchmark, we pointed out each piece and assembled until it came to the final shape. After making the final assembly with all the benchmarks, it is necessary to check the existence of the interferences in the structure, Figure 3, because the presence of interference (overlapping or gaps) can cause the analysis program not to run, or, worse, run with incorrect results [8]. 


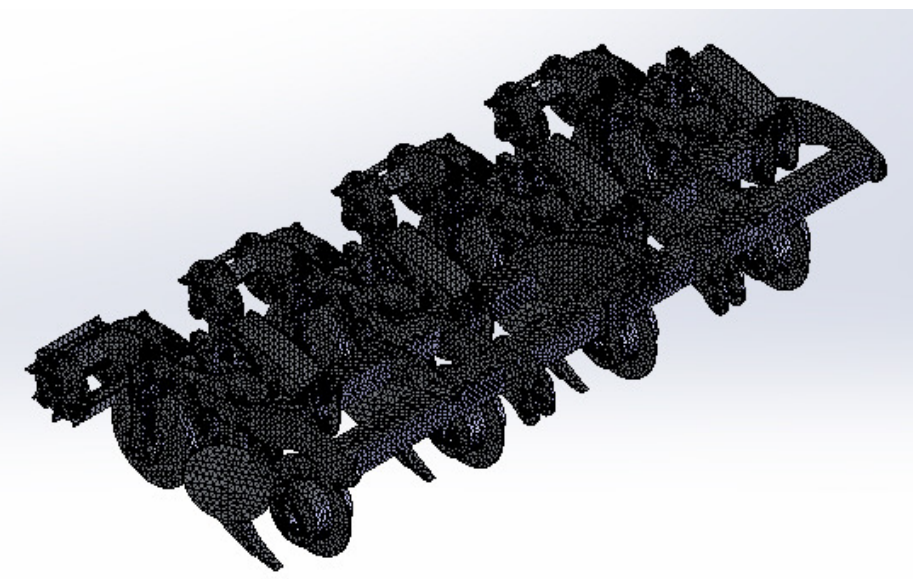

Fig. 2. Meshing of equipment undergoing simulation

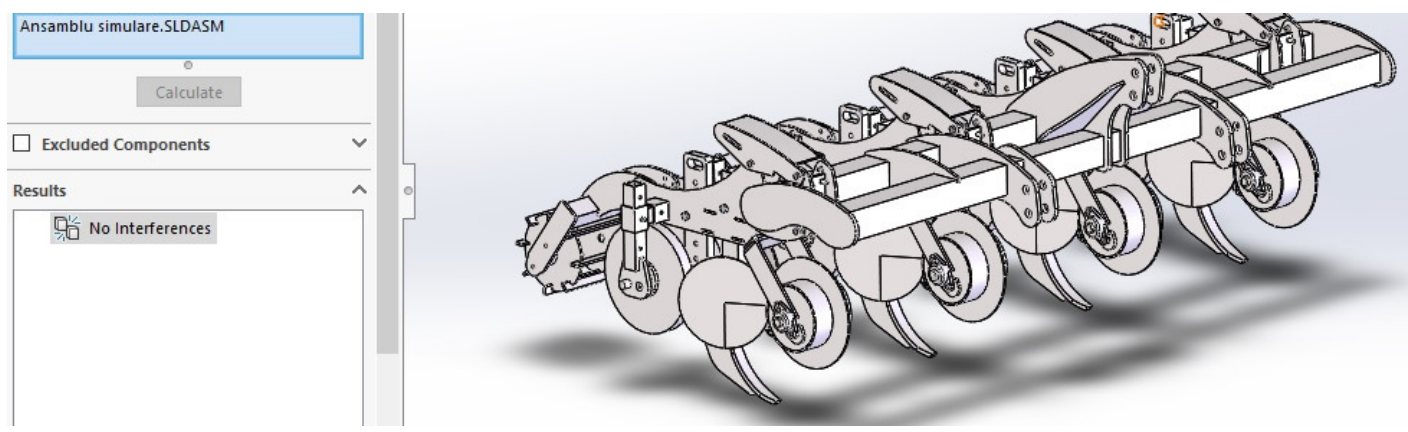

Fig. 3. Checking existence of interferences in structure

In this article we study the structure response only for the maximum normal working load. The total force applied normally to the surface of the working parts projected on the normal plane to the direction of travel was calculated above and resulted in a force of $5600 \mathrm{~N}$.

The S275JR material used for the components of the analyzed structure is shown in table 2, as well as its properties.

Table 2

\section{Properties of S75JR material}

\begin{tabular}{|c|c|c|}
\hline Property & Value & Units \\
\hline Elastic modulus & $2.1 \cdot \mathrm{e}^{11}$ & $\mathrm{~N} \cdot \mathrm{m}^{-2}$ \\
\hline Poisson's ratio & 0.28 & $\mathrm{~N} \cdot \mathrm{A}$ \\
\hline Shear modulus & $7.9 \cdot \mathrm{e}^{10}$ & $\mathrm{~N} \cdot \mathrm{m}^{-2}$ \\
\hline Mass density & 7800 & $\mathrm{~kg} \cdot \mathrm{m}^{-3}$ \\
\hline Tensile strength & $410 \cdot 10^{6}$ & $\mathrm{~N} \cdot \mathrm{m}^{-2}$ \\
\hline Yield strength & $275 \cdot 10^{6}$ & $\mathrm{~N} \cdot \mathrm{m}^{-2}$ \\
\hline $\begin{array}{c}\text { Thermal expansion } \\
\text { coefficient }\end{array}$ & $1.1 \cdot 10^{-5}$ & $\mathrm{~K}$ \\
\hline Thermal conductivity & 14 & $\mathrm{~W} \cdot(\mathrm{m} \cdot \mathrm{K})^{-1}$ \\
\hline Specific heat & 440 & $\mathrm{~J} \cdot(\mathrm{kg} \cdot \mathrm{K})^{-1}$ \\
\hline
\end{tabular}

After the structural analysis, the modal analysis was carried out by following the previous steps, only the load was pure gravitational (its own weight), the gravitational acceleration having the value $9.81 \mathrm{~m} \cdot \mathrm{s}^{-2}$, on the only non-zero component, the one according to the Oy axis.

The structure analyzed in this article is a modern structure of equipment for soil processing in strips, with a load bearing structure made of plates. The modal analysis or frequency analysis for this structure aims at determining the own frequencies and identifying the components with maximum amplitude on each of its own basic frequencies. 


\section{Results and discussion}

\subsection{Structural analysis of the equipment for soil processing in strips}

The main results of linear-elastic static structural analysis are: the values of the reactions in the supports, the distribution of the vector field of the relative-resultant displacement in the structure, the distribution of the tensor fields of the specific deformation and the Cauchy tension in the same structure. Also, an important result for the structure safety is the distribution of the safety factor.

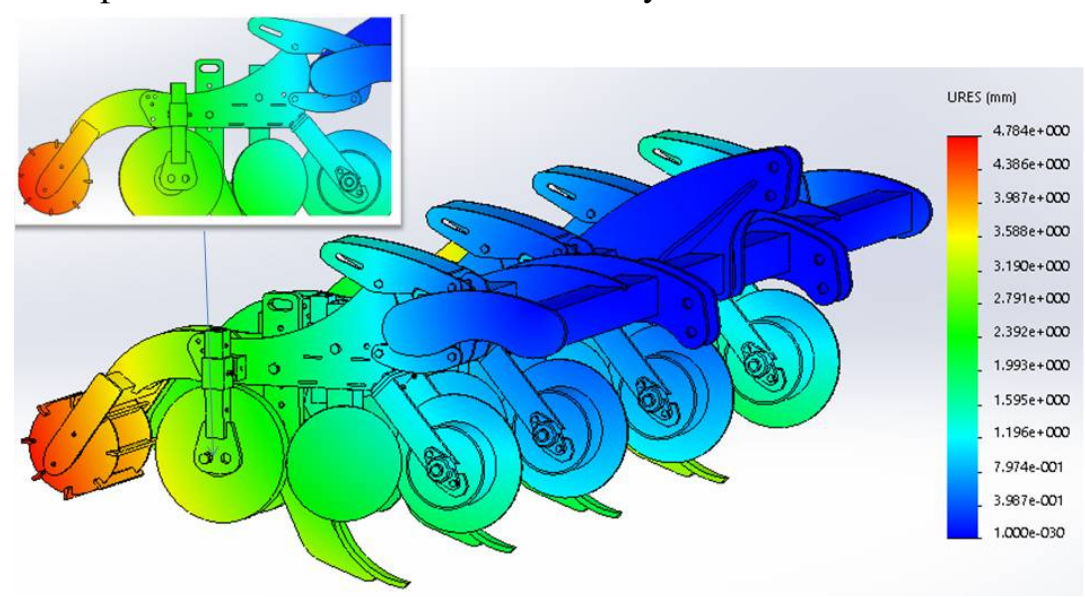

Fig. 4. Distribution of relative displacement field values resulting on structure surface

In Figure 4 the distribution maps of the relative displacement field values resulting on the structure surface are graphically represented. Note that the maximum value (about $4.7 \mathrm{~mm}$ ) is located at the rear of the structure. This maximum value may be exceeded, if we take into account the backlash of the structure and of the connection system between the cultivator and the tractor. Increasing the movement, in the conditions of the considered stress, by admitting the backlash, contributes to the relaxation of the structure and implicitly to the increase of the safety factor. However, exaggerated backlash generally leads to more or less premature wear.

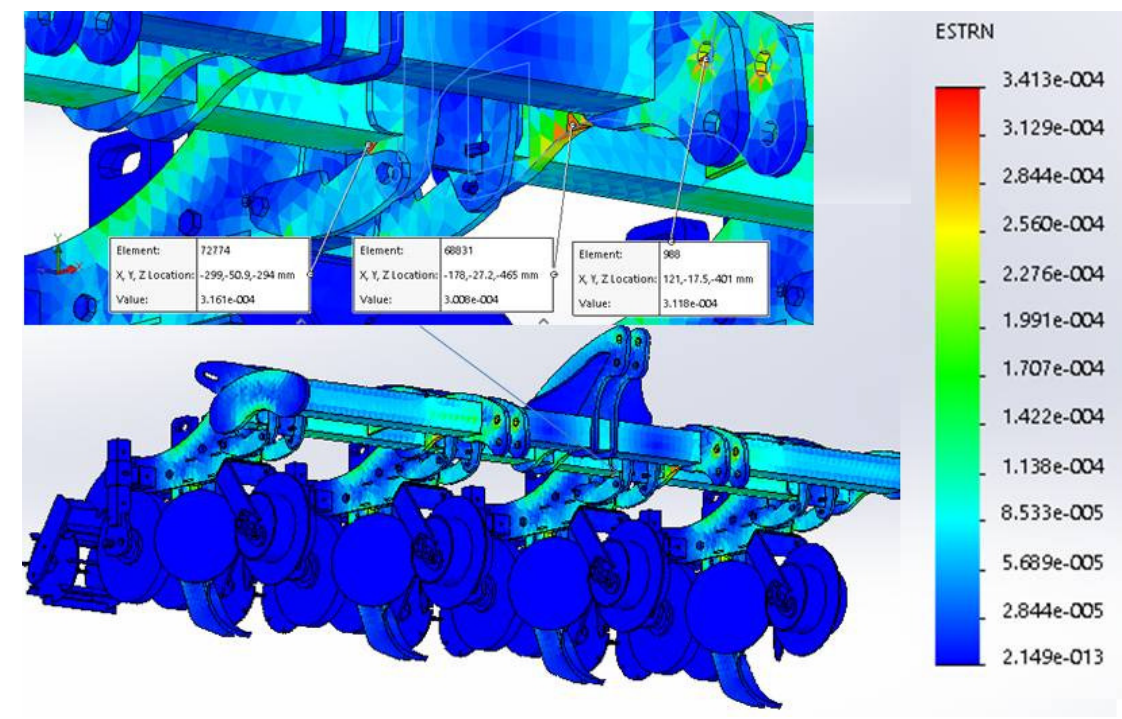

Fig. 5. Distribution of total specific deformation field values on structure surface

In Figure 5, the distribution of the total specific deformation values is graphically represented by the colour map. The area of maximum stress is also highlighted in detail. Due to the fact that we are working in the elastic-linear domain, the maximum tension will be located in the same area as the maximum specific deformation. The maximum equivalent tension is similarly represented graphically in Figure 6 [9]. 


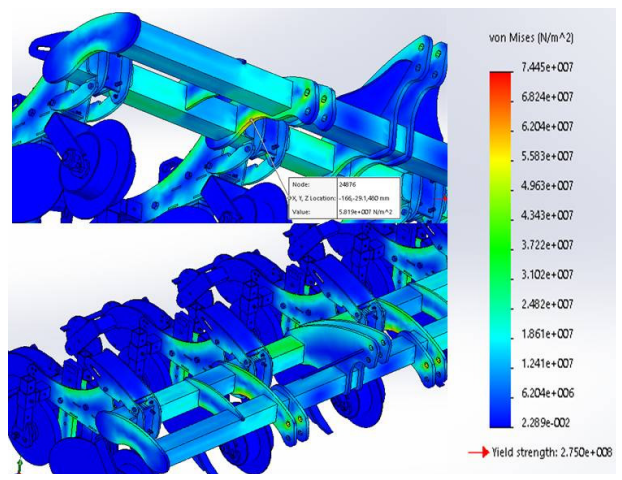

Fig. 6. Representation of equivalent tension distribution on structure surface

Finally, Figure 7 shows the graphical representation of the safety factor distribution in the structure.

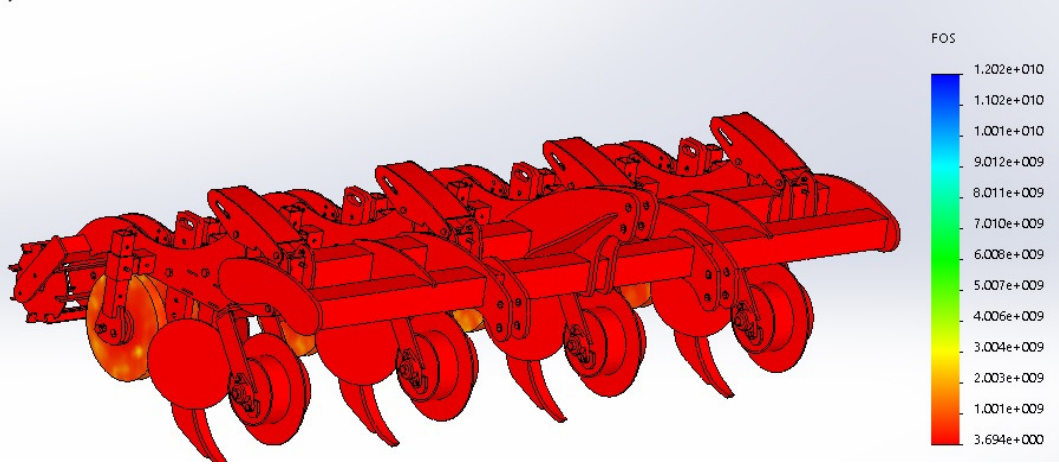

Fig. 7. Safety factor distribution on structure surface

The minimum value of the safety coefficient is 3.6. For agricultural machinery for soil works, the usual safety coefficient values are between 1.8 and 2.2. This machine, therefore, is oversized or works under much tougher conditions. The latter situation can appear either due to use under improper conditions, or due to accidents (impact with hard rocks or roots in the soil) [10].

\subsection{Modal analysis of the equipment for soil processing in strips}

The result of calculating frequencies or modal analysis relies essentially in the list of a number of its own frequencies (in $\mathrm{Hz}$ ) (pulsations, in $\mathrm{rad} / \mathrm{s}$ and periods, in s), in ascending order, starting with the lowest (fundamental frequency). There are presented also the relative displacements on the directions and resultants in the structure for each vibration mode separately. Also, colour maps of the field of relative displacements, on component or resultant, are presented.

In Table 3, it is shown, from the SOLIDWORKS program report, which was used (the SIMULATION module) for making the frequency analysis, a list of the first twenty own frequencies, corresponding to twenty vibration modes, each of them with normalized displacements on the three directions.

Table 3

Mass Participation (Normalized)

\begin{tabular}{|c|c|c|c|c|}
\hline Mode Number & Frequency(Hertz) & X direction & Y direction & Z direction \\
\hline 1 & 12.486 & 0.0023804 & 0.019365 & 0.08436 \\
\hline 2 & 13.107 & 0.059745 & 0.471 & 0.0040362 \\
\hline 3 & 15.655 & 0.00036457 & $9.7261 \mathrm{e}-0005$ & 0.48823 \\
\hline 4 & 17.346 & 0.041143 & 0.018868 & 0.0066403 \\
\hline 5 & 19.513 & $4.2023 \mathrm{e}-005$ & $2.4606 \mathrm{e}-007$ & 0.1277 \\
\hline 6 & 19.942 & 0.0041343 & 0.0013771 & $8.2069 \mathrm{e}-005$ \\
\hline 7 & 23.123 & 0.011566 & 0.092718 & $1.4616 \mathrm{e}-005$ \\
\hline 8 & 25.375 & $1.509 \mathrm{e}-007$ & $5.0515 \mathrm{e}-009$ & 0.0029329 \\
\hline
\end{tabular}


Table 3(continued)

\begin{tabular}{|c|c|c|c|c|}
\hline Mode Number & Frequency(Hertz) & X direction & Y direction & Z direction \\
\hline 9 & 33.652 & 0.019489 & 0.00092254 & 0.0021201 \\
\hline 10 & 34.299 & 0.021748 & 0.0016269 & 0.0021418 \\
\hline 11 & 37.219 & 0.000397 & 0.00029091 & $8.2683 \mathrm{e}-006$ \\
\hline 12 & 37.606 & $1.7165 \mathrm{e}-005$ & $1.0198 \mathrm{e}-008$ & 0.0013906 \\
\hline 13 & 39.64 & 0.063056 & 0.022034 & 0.014833 \\
\hline 14 & 40.04 & 0.2144 & 0.078291 & 0.0042997 \\
\hline 15 & 49.429 & $2.873 \mathrm{e}-005$ & 0.00071347 & 0.015895 \\
\hline 16 & 50.327 & $1.0148 \mathrm{e}-005$ & $2.3651 \mathrm{e}-005$ & 0.029959 \\
\hline 17 & 51.04 & 0.0044104 & 0.0017016 & $4.3454 \mathrm{e}-005$ \\
\hline 18 & 51.102 & $1.8066 \mathrm{e}-005$ & $7.1795 \mathrm{e}-005$ & $2.7716 \mathrm{e}-005$ \\
\hline 19 & 51.188 & 0.0088236 & 0.0053878 & $9.0719 \mathrm{e}-006$ \\
\hline 20 & 51.221 & 0.021435 & 0.012282 & 0.00086952 \\
\hline- & - & Sum X=0.47321 & Sum Y=0.72677 & Sum Z $=0.78559$ \\
\hline
\end{tabular}

Another way of showing these results appears in figures 8,9,10. This figures presents own frequencies and maps of the amplitudes on the deformed shape of the structure, in the vibration modes corresponding to the respective frequency. In this figures, only three of the twenty calculated vibration modes are given for reasons of fitting into a certain number of pages of an article.

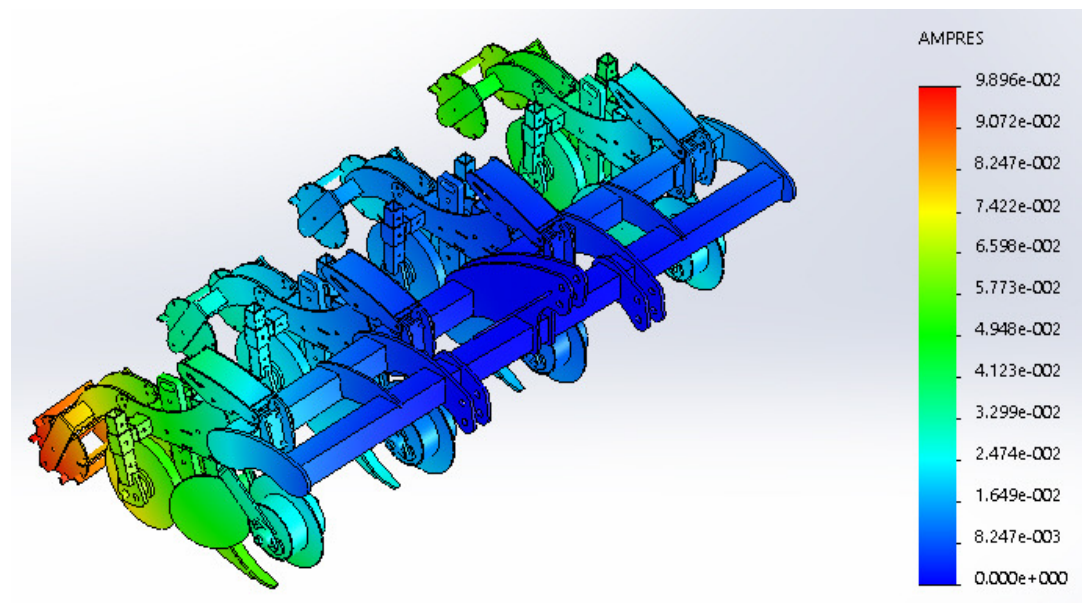
Fig. 8. The $1^{\text {st }}$ frequency of the structure $\mathbf{1 2 . 4 8 6}$ and the amplitude maps on its surface, in case
of oscillation on the respective own frequency

The results obtained and provided by modal analysis for mechanical structures are relatively few and their uses are accurate.

As it is known, the main result of the modal analysis is the set of calculated frequencies. In principle, we can ask the program to calculate an unlimited number of own frequencies. In fact, just the first few are useful. The most important is the fundamental frequency, which has the lowest value of the calculated ones. Most of the time, the list of own frequencies is used to avoid resonance working regimes and, in general, resonance phenomena that may occur under various circumstances.

For the structure analyzed in this paper, we limited the number of frequencies calculated to the first twenty. We considered that this way we cover all the basic frequencies that may occur in the equipment working process. The highest frequencies we had in view were those that usually come from the tractor engine $(33-67 \mathrm{~Hz})$, although through the coupling mode between the cultivator and tractor, the transmission of these frequencies from the tractor to the cultivator is highly unlikely to amplitude that is noticeable by the usual measurement and control equipment. 


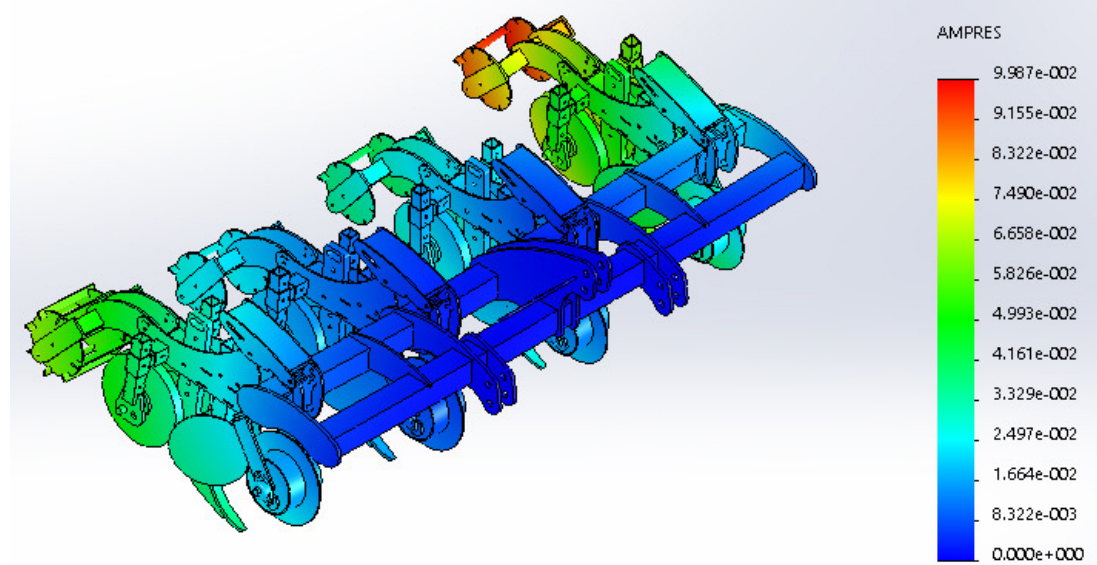

Fig. 9. The $2^{\text {nd }}$ frequency of the structure 13.107 and the amplitude maps on its surface, in case of oscillation on the respective own frequency

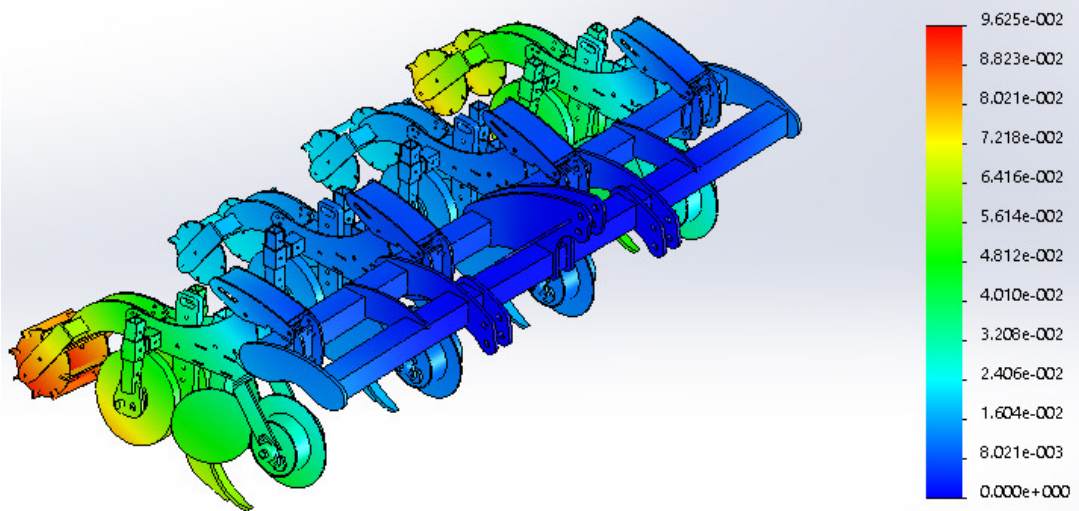

Fig.10. The $3^{\text {rd }}$ frequency of the structure $\mathbf{1 3 . 1 0 7}$ and the amplitude maps on its surface, in case of oscillation on the respective own frequency

On the other hand, we tried to cover the frequency spectrum that could affect the tractor operator's health, according to the standards. [11] Affecting the operator by the cultivator own frequencies is also unlikely, as the periodic signals transmitted from the cultivator to the tractor are weak in intensity also due to the coupling mode and attenuated in the tractor suspension system (including wheels for wheeled tractors). Concerns on the ergonomic line remain in place and receive new dimensions [12].

\section{Conclusions}

Following this first structural study on the cultivator for soil processing in strips, several important conclusions can be drawn for further investigations.

For a normal stress, calculated according to the criteria outlined in the paper, the cultivator load bearing structure is overestimated, without taking into account potential accidents in the soil (hard bodies) or use under improper conditions.

Considering the equivalent tension maximum values, there is no danger of failure of the structure material. Also, the maximum values of resultant relative displacement guarantee that deviations from the working parameters indicated by agrotechnical requirements (working depth, in particular) are negligible, at least at the essential working part of the cultivator.

The value of the safety coefficient (3.7), in relation to its usual values in the practice of designing and manufacturing of agricultural machines for soil tillage, shows that there is a potential for optimization of this equipment. Because the scarifier could be used in soil that was untilled for five to 
ten years, there is the possibility of accepting a coefficient of up to $7-8$, therefore, it is necessary to make a substantial optimization study.

The analysis of the cultivator own spectrum allows identifying quite accurately the main frequencies, on which a resonant working regime can occur. The fundamental frequency of the analyzed structure is $12,486 \mathrm{~Hz}$. This is an unreachable frequency under normal working conditions, where the tractor does not induce dangerous excitations. For example, a tractor with a wheel diameter of about $1.75 \mathrm{~m}$ should have 8 profiles on circumference that would emit an excitation on the $11.5 \mathrm{~Hz}$ frequency, which could cause resonance in the scarifier structure.

Regarding the possibility of generating oscillations on own frequencies to be transmitted through the coupling system to the tractor and implicitly to the tractor operator, although the phenomenon is very unlikely, in terms of dangerous frequencies existence, the danger exists. For example, modes from 7 to 20 have frequencies within the range that can affect the eyeball, the frequencies corresponding to modes 16 to 20 are within the range of frequencies affecting the chest, the basic frequency being in the range that affects the spine. Oscillations in modes 9-20 are made on frequencies that can affect the hands. Oscillations in modes 4 and 8 (with frequencies closest to the fundamental frequency may affect the forearm). These statements are made using Table 1 of this paper and the map of human body areas affected by oscillations on certain frequencies.

\section{Acknowledgements}

The work was supported by a grant of the Romanian Ministry of Research and Innovation, CCCDI-UEFISCDI, project number PN-III-P2-2.1-CI-2017-0151, within PNCDI III.

\section{References}

[1] Letosnev M. N. Agricultural Machines, Agrosilvica State Publishing House, Bucharest, 1959

[2] Cardei P., Georgi K., Working regimes of the agricultural machines designed to soil tillage: From optimization to fundamentals (2), INMATEH, vol. 37, No. 2/2012, pp. 21-28.

[3] Mahmood H. F., Subhi Q. A., Hussein E. K., Comparison of Vibrations, Tillage Depths and Soil Properties for Moldboard and Disk Plows at Three Tillage Speeds, Asian Journal of Agricultural Research 5 (1), 2011, pp. 90-97,.

[4] Ragni L., Vassalini G., Xu F., Zhang L. B., Vibration and Noise of Small Implements for Soil Tillage, Journal of Agricultural Engineering Research, Vol. 74, Issue 4, 1999, pp. 403-409.

[5] Karoonboonyanan R., Salokhe V.M., Niyamapa T., Nakashima H., Vibration Effects on the Performance of a Single-Shank Subsoiler, Agricultural Engineering International: the CIGR Ejournal. Manuscript PM 07 018. Vol. IX. September, 2007.

[6] Matache M., Voicu Gh., Cardei P., Vladut V., Persu C., Voicea I., Accelerated test of MAS 65 deep soil loosening machine frame, Proceedings of the 43rd international symposium on agricultural engineering, Opatija, Croatia, 2015, pp. 131-139

[7] Sandru A., Popescu S., Cristea I., Neculaiasa V., Operation of agricultural machinery, Didactic and pedagogical publishing house, Bucharest, 1983

[8] Krasnicenko A.V. Handbook of AGRICULTURAL MACHINES, volume 2, Technical Publishing House Bucharest, 1964.

[9] Cardei P., Meca A., Kostadinov G., Working regimes of the agricultural machines designed to soil tillage: From optimization to fundamentals (1), INMATEH, vol. 37, No. 2, 2012, pp. 13-20.

[10] David A., Voicu Gh., Marin E., Dutu M., Gheorghe G., Experimental researches on working qualitative indexes of a deep loosening equipment, INMATEH, vol. 46, no. 2, 2015, pp 5-12.

[11]Bruel \& Kjaer, Human Vibration, [online] [November 1989] Available at: https://www.bksv.com/ /media/literature/Primers/br056.ashx?la = en

[12] Futatsuka M., Maeda S., Inaoka T., Nagano M., Shono M., Miyakita T., Whole-Body Vibration and Health Effects in the Agricultural Machinery Drivers, Industrial Health, Vol. 36, No. 2, 1998, pp. 127-132. 